\title{
FIN DE VIE : LE REGARD ITALIEN
}

\author{
Laurence KLESTA*
}

À mon père

Le projet de loi italien envisage les situations de fin de vie dans le cadre de la relation de soins caractérisée par l'équilibre entre les compétences du médecin et du patient, le principe d'auto-détermination de ce dernier et l'utilité thérapeutique. Si celleci fait défaut, le projet, prenant appui sur la Constitution, affirme le droit, pour toute personne, d'établir le moment où le processus naturel de la mort doit reprendre son cours. Lorsque la mise en œuvre de ce droit s'inscrit dans la durée, le refus de soins est appréhendé par des critères de sécurité juridique ; à défaut, la recherche de la volonté du patient emprunte les valeurs propres à l'harmonie du vécu afin de sauvegarder, en toutes circonstances, la dignité de la personne. L'originalité du texte tient à la capacité d'écoute individuelle de la règle à l'égard de situations qui, par hypothèse, sont intemporelles et transcendent le domaine juridique.

The Italian bill considers end-of-life situations in the framework of health care relationship characterized by balance of skills between doctor and patient, respect for patient autonomy and effective treatment. When burden outweighs any benefit, the personal right to decide when dead process should return to normal, is clearly embodied. If that choice has been previously contemplated, treatment refusal is dealt with through legal rules; failing that, life story criteria act as guidelines to enforce patient's right and safeguard, in any circumstances, his dignity. The Italian main feature's lies in the rule's listening capacity with regard to situations, which, by definition, are timeless thereby going beyond law.

${ }^{*}$ Ricercatrice, Dipartimento di diritto privato e critica di diritto, Université de Padoue. 
C'est sur la volonté du patient que se fonde le projet de loi italien ${ }^{1}$ pour élaborer un cadre aux situations de fin de vie ${ }^{2}$. Face à cette question aux multiples aspects - biologique, médical, idéologique, social ${ }^{3}$ - que représente le moment ultime, le texte organise, au sein de la relation de soins, un espace décisionnel à la personne pour qu'elle en rythme la mesure et le temps : se décline ainsi un « droit gentil » ${ }^{4} \ldots$ qui ne peut faire de $\mathrm{mal}^{5}$.

Le refus de tout traitement médical sur lequel il repose, suppose l'acceptation d'une évolution naturelle vers la mort à l'égard de laquelle la norme sociale n'a que peu de prise, ne pouvant s'inscrire dans la durée ${ }^{6}$.

${ }^{1}$ Le projet de loi (ci-après projet) portant dispositions en matière de relation de soins, consentement, urgence médicale, refus et interruption des soins, déclarations anticipées » a été communiqué à la présidence du Sénat le 15 mars 2013 ; le document est consultable sur le lien suivant : http://www.senato.it/service/PDF/PDFServer/BGT/00720602.pdf; en général sur la procédure législative, v. le site du Sénat italien : http://www.senato.it/leg/17/BGT/Schede/ Ddliter/39310.htm. Ce projet est le fruit d'un travail collectif, objet d'un colloque « Per un diritto gentile in medicina " présidé par le professeur Paolo Zatti dont la synthèse est publiée : https://undirittogentile.wordpress.com/2013/11/21/atti-del-convegno-per-un-diritto-gentile-inmedicina-padova-25-ottobre-2013/. Le projet est proche de la loi allemande de 2009 Patientenverfügung en raison des affinités qui caractérisent les droits allemand et italien.

Quant à l'état des lieux français, la proposition de loi modifiant la loi Leonetti relative aux droits des malades et à la fin de vie (2005-370) a été définitivement adoptée le 27 janvier 2016, mettant ainsi un terme au désaccord persistant entre l'Assemblée nationale et le Sénat. La loi «créant de nouveaux droits pour les personnes malades en fin de vie » composée de quatorze articles, modifiera le Code de la santé publique, en particulier les articles 1110-5, 1111-4, 1111-6, $1111-11,1111-12$ et 1412-1-1. La version provisoire à ce jour (29 janvier 2016) ainsi que les différentes étapes de la procédure législative sont consultables sur le lien suivant : http://www.senat.fr/espace presse/actualites/201504/fin de vie creer de nouveaux droits en fave ur des malades.html\#c616069; sur la loi Leonetti, v. le colloque organisé par l'Université de Lille «La fin de vie saisie par le droit : le droit de la fin de vie »: http://www.conseiletat.fr/Actualites/Discours-Interventions/Les-10-ans-de-la-loi-Leonetti-le-droit-de-la-fin-de-vie.

${ }^{2}$ Les situations de fin de vie sont celles «dans lesquelles l'altération grave de l'état de santé en raison de la progression d'une maladie ou pour une autre raison engage le pronostic vital d'une personne dans un avenir proche », v. Conseil de l'Europe, Guide sur le processus décisionnel relatif aux traitements médicaux dans les situations de fin de vie, p. 8.

${ }^{3}$ M. CAULI, "Comprendre la fin de vie : représentations sociales et culturelles », in adps, Penser et vivre la fin de vie, déc. 2014, p. 14.

${ }^{4} \mathrm{Ce}$ « droit gentil » fait référence aux discussions et recherches préliminaires qui ont entouré le projet, v. supra note 1 . Il ne renvoie pas à un droit mou mais à un droit qui, par référence à l'étymologie (gens : communauté familiale élargie/société civile) est caractérisé par son objectif: «promouvoir une relation de soins digne d'une société et d'une médecine gentilles» et son approche : "prendre soin des problèmes réels », v. P. ZATTI, «Per un diritto gentile in medicina », in Per un diritto gentile alla fine della vita, cit. supra note (1). D'où un droit sensible, adapté à la personne.

${ }^{5}$ Ibid.

${ }^{6}$ L'homme ne pouvant dominer une donnée qui lui échappe (le moment de la mort), le droit, comme construction sociale peut, au mieux, organiser le temps présent, v. à ce propos la belle étude de C. CHAINAIS, Cour de cassation, Rapport annuel 2014, Étude - Le temps, Avant-propos : https://www.courdecassation.fr/publications_26/rapport_annuel_36/rapport_2014_7040/livre_3_etu de_temps_7047/avant_propos_31865.html. 
L'euthanasie et le suicide assisté ${ }^{7}$ ne sont pas envisagés puisqu'ils consistent à hâter la fin de vie ${ }^{8}$ et qu'ils ne relèvent pas de la culture du soigner ${ }^{9}$ au sein de laquelle se place résolument le projet. L'accès aux soins palliatifs, essentiel au processus d'accompagnement, est depuis 2010, l'objet d'une loi spécifique $^{10}$.

Fort d'un climat constitutionnel favorable aux droits de la personne, le corpus juridique construit au fil des ans par la jurisprudence italienne, est précisé et intégré pour concrétiser le principe de dignité dans les décisions et traitement de fin de vie : sauvegarder la qualité de vie du patient en lui permettant de valoriser, à l'extrême, son identité et réintégrer ainsi le temps qui lui reste à vivre ${ }^{11}$.

En toile de fond se pose la question de la place du droit - minimale mais nécessaire - dans la régulation d'une situation complexe et incertaine, et celle de son application à une société « humaine » qui saurait appréhender l'inéluctable en harmonie avec le vécu : retrouver, à la limite du temps, le logos de la proportion ${ }^{12}$.

${ }^{7}$ Alors que l'euthanasie est l'acte intentionnel de donner la mort en accord avec le malade, le suicide assisté consiste en une auto-administration d'une dose létale avec le recours d'un tiers, v. adps cit., p. 17.

${ }^{8}$ L'euthanasie et surtout le suicide médicalement assisté (qui révèle une exigence d'autonomie par rapport à la médecine) renvoient à une vision utilitariste de l'existence où la fin de vie est considérée comme chronophage, c'est-à-dire qu'elle fait perdre du temps (v. B. MORIZOT, « Enjeux philosophiques du suicide médicalement assisté » : adps cit., p. 21), c'est-à-dire une vision antithétique par rapport au projet italien.

9 V. en particulier l'art. 32 de la Constitution italienne relatif à la santé : «La République protège la santé en tant que droit fondamental de l'individu et intérêt de la collectivité, et garantit aux indigents des soins gratuits ».

${ }^{10}$ La loi du 15 mars $2010, \mathrm{n}^{\circ} 38$ portant « dispositions pour garantir l'accès aux soins palliatifs et à la thérapie contre la douleur » qualifie de tels soins de " niveaux essentiels d'assistance », c'està-dire de prestations minima que l'État et les régions doivent assurer sur tout le territoire. La loi s'inscrit dans le cadre d'une approche favorable aux droits de la personne qui suppose la prise en charge de la dignité et de l'autonomie des patients et est opposée à tout acte qui provoquerait la mort. C'est de même la philosophie qui anime le projet sur la fin de vie dont le fondement est la volonté de la personne. Pour une vue d'ensemble de la loi $n^{\circ}$ 2010/38, v. le site du ministère de la Santé : http://www.salute.gov.it/portale/temi/p2_4.jsp?area=curePalliativeTerapiaDolore. Sur la situation française en matière de soins palliatifs, v. V. MOREL, « Les soins palliatifs depuis 20 ans : de la prise de conscience de leur nécessité à la gestion des situations complexes de fin de vie », in adps cit., p. 40.

${ }^{11}$ C'est l'idée de réconciliation de soi mise en avant par P. Ricœur, v. A. FORNES, P. BONHOMME, «Le patient, la famille et les soignants face au travail de deuil de fin de vie », in adps cit., p. 19.

${ }^{12}$ Le logos, ou raison déterminant le monde, fait référence au concept d'harmonie et à ses critères de base que sont la proportion et l'équilibre, v. P. ZATTI, « Diritto e medicina in dialogo : il logos della proporzione », in Un diritto gentile cit. note (1). 
Formée de vingt-cinq articles regroupés en six chapitres ${ }^{13}$, le projet de loi jette les bases d'une gouvernance de la relation de soins puis modèle la règle pour qu'elle reflète un choix identitaire : le droit devient « agile ».

\section{RELATION DE SOINS ET AUTONOMIE DÉCISIONNELLE}

Deux valeurs essentielles, complémentaires l'une de l'autre, servent de support à la relation de soins ${ }^{14}$ envisagée comme paradigme d'une société

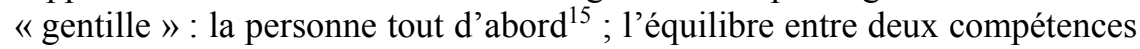
ensuite, celle du patient vis-à-vis de lui-même et celle du médecin sur le processus de santé.

$$
\text { A. }- \text { Un rapport « personnel } »^{16}
$$

\section{La personne du patient : une symbiose de droits fondamentaux}

La relation de soins est envisagée comme le support de droits fondamentaux. Sont ainsi proclamés l'intangibilité du corps humain ${ }^{17}$ et le droit à «poursuivre et atteindre un état de santé physique, psychique et relationnel selon son vécu et sa conception de la vie $»^{18}$. À ce cadre « subjectivisant », en ce sens qu'il est fondé sur les droits subjectifs ${ }^{19}$, se rattache également la référence à l'habeas corpus, symbole de liberté

\footnotetext{
${ }^{13}$ Chapitre I Droit sur son corps et droit à la santé - Chapitre II Relation de soins - Chapitre III Consentement au traitement - Chapitre IV Refus de soins - Chapitre V Urgence médicale Chapitre VI Dispositions anticipées de traitement.

${ }^{14}$ En droit italien, la relation de soins est contractuelle quelle que soit la nature privée ou publique de l'établissement de soins. Mise en œuvre du droit fondamental à la santé, ce modèle, symbole de confiance, est plus protecteur des intérêts du patient notamment au niveau de la charge de la preuve. La conclusion du contrat atypique de spedalità intervient au moment de l'acceptation, par l'établissement de soins, du patient, v. L. KLESTA, «La responsabilité médicale en Italie : le renouveau du « contrat social »? », in RIDC n ${ }^{\circ} 2012-3$, p. 757.

15 À cet égard, on peut déplorer que la proposition de loi française définitivement adoptée le 27 janvier 2016 ait pour objet les droits des « personnes malades en fin de vie » non seulement car la fin de vie ne concerne pas seulement les malades mais aussi parce que l'adjectif « malade » affaiblit l'idée d'humanité inhérent le concept de personne, c'est-à-dire sa vraie valeur.

${ }^{16}$ Le rapport étant, par hypothèse, une relation entre - au moins deux - personnes, c'est à travers l'emploi de l'adjectif «personnel » qu'est transposée l'idée de confiance, voire d'intimité, qui caractérise la relation de soins.

${ }^{17}$ Projet, art. 1. $1^{\mathrm{er}}$ al.

${ }^{18}$ Projet, art. 2.

${ }^{19}$ Projet, art. 4.
} 
personnelle ${ }^{20}$ et refuge des principes de dignité, intégrité, identité et autodétermination applicables en la matière.

Le projet pose ainsi les bases de la " gouvernance du corps » (governo del corpo) forgée par la doctrine à partir des articles 2,13 et 32 de la Constitution $^{21}$, transposant la conception philosophique selon laquelle le corps n'existe qu'à travers la personne. Cette dernière, être doué de conscience, est titulaire du droit à choisir «sa » santé qui comprend deux aspects essentiels : la liberté de l'individu sur lui-même, toute atteinte au corps d'autrui n'étant licite que si l'intéressé (ou son représentant) y consent $^{22}$; et l'exercice de cette liberté, à savoir l'accès à l'information thérapeutique. Le patient n'est libre que s'il dispose d'un espace décisionnel qui lui permet de choisir en toute connaissance de cause les soins les plus appropriés à son état.

Ces deux profils recoupent la notion d'autonomie de fait - capacité à prendre les bonnes décisions pour $\operatorname{soi}^{23}$ - et fondent celle de compétence ${ }^{24}$ à la base de la définition de la santé adoptée par l'OMS ${ }^{25}$ : état physique et mental, mais surtout rapport ayant pour objet le « bien » de l'une des parties, c'est-à-dire le bénéfice procuré par les traitements médicaux.

Ce bénéfice n'est autre que la mise en œuvre du principe de proportion. La relation de soins ne peut en effet être telle qu'au vu de son utilité thérapeutique, c'est-à-dire qu'elle doit justifier une perspective de stabilisation et/ou d'amélioration des conditions de santé du patient; dès

\footnotetext{
${ }^{20}$ Dirigée contre l'arbitraire du pouvoir qui sévissait dans l'Angleterre médiévale, la procédure de l'habeas corpus, introduite dès 1215 dans la Magna Carta, devint une loi du Royaume en 1679. Littéralement l'expression «Que tu aies ton corps » signifie que te soit redonnée la liberté physique.

${ }^{21}$ Selon l'art. 2 de la Constitution (1948) : «La République reconnaît et garantit les droits inviolables de l'homme, comme individu et comme membre de formations sociales où s'exerce sa personnalité, et exige l'accomplissement des devoirs de solidarité politique, économique et sociale auxquels il ne peut être dérogé ». L'art. $13 \mathrm{C}$ énonce le principe de l'inviolabilité de la liberté personnelle et l'art. $32 \mathrm{C}$ proclame le droit fondamental à la santé et le droit au refus des traitements médicaux obligatoires. Sur cette base, la Corte costituzionale a pu élaborer une jurisprudence étoffée grâce à la procédure du controllo di costituzionalità in via incidente (équivalent de la question prioritaire de constitutionnalité) prévue à l'art. 134, al. 1, C; ainsi fut reconnue à tout patient « la liberté de disposer de son corps dans tous les domaines où trouve expression la personne humaine », v. Corte cost., 22.10.1990, n. 417.

${ }^{22}$ Projet, art. $1,2^{\mathrm{e}}$ al.

${ }^{23}$ A. FORNES, P. BONHOMME, cit., p. 21.

${ }^{24}$ Projet, art. 4, al. $2:$ « ... la compétence du patient à déterminer son propre « bien » selon ses convictions, expériences et préférences ».

${ }^{25}$ Selon le Préambule à la Constitution de l'Organisation mondiale de la santé, adopté par la Conférence internationale sur la santé, New-York, 19-22 juin 1946, «La santé est un état de complet bien-être physique, mental et social, et ne consiste pas seulement en une absence de maladie ou d'infirmité ».
} 
lors tout prolongement «matériel » de la survie doit être considéré comme une obstination déraisonnable ${ }^{26}$.

Ce souci d'équilibre, cette proportion en termes de coût et de bénéfice (au sens large) est l'objet d'un droit fondamental consacré dans le projet italien : celui de toute personne, qui échappe au savoir médical, d'établir le moment où le processus naturel de la mort doit reprendre son cours ${ }^{27}$. Il s'agit de l'autonomie.

L'article 4 pose le principe selon lequel le consentement de la personne est « condition essentielle du choix, du début et de la poursuite des soins». La compétence du patient s'inscrit ainsi au sein d'un parcours décisionnel, au contenu variable, mais dont l'essence est, et reste le choix exprimé par le patient. Partant, sa volonté peut se manifester sous de multiples formes: support écrit mais également enregistrement audio ou vidéo ${ }^{28}$; de même, par souci d'intégrité et afin de suppléer les hypothèses d'incapacité civile, différentes modalités d'accompagnement et/ou de substitution du consentement, en particulier en cas de refus ou de renoncement aux soins en cours de traitement ${ }^{29}$, sont envisagées.

À cet égard, le projet de loi ne prescrit pas de règle de conduite générale et abstraite, mais adapte, à une situation individuelle, des critères de sécurité juridique ${ }^{30}$ : qu'il s'agisse de la possibilité d' « indiquer» ou de « nommer » une personne de confiance ou encore de valoriser l'exercice du droit à l'autodétermination ${ }^{31} \mathrm{du}$ mineur ou de l'incapable majeur ${ }^{32}$. La démarche n'en est pas moins normative en ce sens qu'elle est prévisionnelle, elle s'inscrit dans une durée, la plus subjective possible. À cette fin, le projet dispose de deux lignes directrices, concrètes et individuelles pour guider le processus décisionnel : l'adaptation aux facultés de compréhension de la

\footnotetext{
${ }^{26}$ P. ZATTI, Premesse e criteri per un diritto della dignità del morire e delle "disposizioni anticipate del paziente" : http://www.giurisprudenza.unipr.it/sites/st07/files/allegatiparagrafo/16-042014/premesse_e_criteri_per_un_diritto_della_dignita_del_morire_e_delle_disposizioni_anticipate_ del_paziente.pdf

${ }^{27}$ Projet, art. 3, al. $1^{\text {er }}$ : « la relation de soins a pour seul but le bien du patient, qui repose sur la collaboration avec le professionnel de santé, en fonction des compétences de chacun, et dont l'ultime détermination revient au patient $»$.

${ }^{28}$ Les manifestations écrites, documentées dans le dossier médical, procès-verbaux, enregistrement audio et vidéo, v. Projet, art. 5.

${ }^{29}$ Projet, art. 6 dans l'hypothèse où la personne, en cours de traitement, ne serait plus en mesure de dialoguer (au sens large) avec l'équipe médicale.

${ }^{30}$ Projet, art. 7, al. 3, par renvoi à 1'art. 4, alinéa 3, prévoit que la personne a droit, en toute circonstance, au respect de tout ce qui caractérise son identité personnelle, qu'il s'agisse de ses besoins, préférences, aspirations, convictions, .... De même, le renvoi à la conception de vie du patient et à tout ce qui constitue son identité, est opéré à l'art. 9 , al. 2 dans l'hypothèse où la personne, incapable majeure, n'a pas désigné de personne de confiance.

${ }^{31}$ Projet, art. 7, al. 1.

${ }^{32}$ Que ce droit se traduise par un consentement ou un refus de soins.
} 
personne et la proportionnalité par rapport à l'enjeu en cause ${ }^{33}$ aussi et surtout en cas de « grave préjudice $»^{34}$.

\section{La personne du médecin : un professionnel de santé}

Parce que la santé est relationnelle et qu'elle se développe dans le cadre d'un établissement de santé (public ou privé) la reconnaissance de droits au profit du patient a pour corollaire, à charge du médecin, l'accomplissement d'obligations correspondantes; en tant que « professionnel de santé », celuici doit agir conformément au principe d'efficacité des soins, ce qui suppose d'interagir avec le patient en le faisant participer aux choix diagnostiques et thérapeutiques ${ }^{35}:$ c'est ce qu'énonce le principe de la consensualita ${ }^{36}$. Cette obligation se conçoit en lien avec la responsabilité individuelle du praticien, qui reste le principe, et auquel ne fait pas obstacle la collégialité de la prise de décision médicale ${ }^{37}$.

Concrètement le médecin, initiateur de la prise de décision, doit « encourager et accompagner le processus de formation du consentement » du patient en tenant compte de sa personnalité ; à cet effet, sont déterminants les critères tenant à l'âge, à la maturité, au niveau culturel et aux conditions de vie de celui-ci. Son identité doit être, en toutes circonstances, sauvegardée ${ }^{38}$.

Le projet de loi reprend les principaux enseignements de la jurisprudence constitutionnelle en matière de consenso informaton $^{39}$ : la

${ }^{33}$ L'art. 7 du projet se réfère à la mesure concrète des capacités de compréhension et de décision du mineur et au caractère adéquat tant des informations, par rapport à l'entendement de la personne, que de l'autodétermination, en fonction de l'importance des décisions (respectivement al. 1,2 et 3). L'art. 8 prend en considération le degré de maturité du mineur pour intégrer ou remplacer sa volonté ; quant à l'art. 9, il vise le cas de l'incapable majeur qui, soit bénéficie d'une protection légale, soit en est privé, auquel cas le professionnel de santé est tenu, si aucune personne de confiance n'est désignée, de consulter toute personne ayant des liens affectifs avec l'incapable afin de témoigner sur les manifestations de volonté/préférences/convictions/conceptions de vie du patient incapable. En cas d'impossibilité, il est fait application de la procédure de protection instituée par la loi 9.01.2004, n. 6 relative à l'amministrazione di sostegno.

${ }^{34}$ Cette hypothèse prévue à l'art. 11 du projet justifie la saisine du juge des tutelles en cas de désaccord entre les personnes habilitées à prendre la décision médicale.

${ }^{35}$ Projet, art. 4, al. 2.

${ }^{36}$ Projet, art. 3, al. 2, v. aussi P. ZATTI, «Consistenza e fragilità dello ius quo utimur in materia di relazione di cura », in Nuova giurisprudenza civile commentata, 2015, p. 20 et s.

${ }^{37}$ Le principe de collégialité de la décision, qui ne subit pas de limitation (alors que le texte français en prévoit, v. amendement 12 à l'art. 2 sur le refus de l'obstination déraisonnable) n'a pas d'incidence à cet égard. La responsabilité individuelle du médecin renvoie - principalement - à la responsabilité pénale. La référence - dans le texte français - à l'obligation de moyens, technique de droit civil, peut ainsi susciter quelques perplexités.

${ }^{38}$ Projet, art. 4, al. 2.

${ }^{39}$ L'expression, pour la première fois employée en jurisprudence en 1985 par analogie à la version anglo-saxonne « informed consent » est actuellement supplantée par celle de « consentement prêté en toute connaissance de cause » (consenso consapevolmente prestato). 
liberté décisionnelle du patient dépend non seulement des informations médicales reçues, ce lien étant la garantie d'un choix conscient et libre ${ }^{40}$; et le rapport de confiance qu'il sous-tend doit être également objectivement fondé car il renvoie aux compétences scientifiques du professionnel de santé et au système de soins, support d'exercice de la profession mais aussi « capteur » d'attention à la personne.

Ce dernier aspect, novateur, se traduit par l'obligation, à charge des établissements de santé, de former leurs personnels afin qu'ils acquièrent les compétences professionnelles pour mieux comprendre les enjeux des situations individuelles. À cet effet, le projet de loi institue des bonnes pratiques et alloue des ressources financières ${ }^{41}$. Il s'agit de mettre en œuvre un choix de société.

\section{B. - Un rapport équilibré}

\section{La programmation "partagée» des soins: la prévision de l'équilibre}

L'équilibre et le processus d'accompagnement qu'il sous-tend ${ }^{42}$ est encouragé. Il reflète l'harmonie du vécu et, s'inscrivant dans la durée, tire profit de l'instrument juridique, symbole de prévision : l'accord des parties en mesure de programmer le futur dès lors qu'il est le fruit d'un " partage ». Cette idée est véhiculée à travers l'adjectif condivisa qui caractérise le pacte passé entre le patient et l'équipe de soins, soulignant ainsi la participation directe de celui-ci à la prise de décision thérapeutique ou indirecte à travers la personne de confiance ${ }^{43}$. Toute forme de paternalisme médical est évidemment rejetée.

L'accord ne peut être écarté qu'en extrême ratio et sous contrôle judiciaire: lorsque, dans l'hypothèse d'incapacité du patient et suite au désaccord persistant - après l'intervention d'experts - entre le responsable des soins et la personne de confiance sur la mise en œuvre du programme de soins, le juge des tutelles est saisi et fait droit à la décision du premier ${ }^{44}$.

\footnotetext{
${ }^{40}$ Ces informations doivent donc être "les plus exhaustives possibles », v. Corte cost., 23.12.2008, n. 438 .

${ }^{41}$ Projet, art. 3, al. 3 ; ainsi de même les articles 1 et 14 de la proposition de loi française définitivement adoptée.

${ }^{42}$ A. FORNES, P. BONHOMME, cit., p. 21.

${ }_{43}$ Projet, art. 17, al. 2: la personne de confiance a le droit de remplacer le «constituant (fiduciante)/professionnel de santé » pour déterminer concrètement le programme de soins et décider en cas de situations imprévues.

${ }^{44}$ Projet, art. 17, al. 3 in fine.
} 
Le contenu de la programmation s'entend des traitements acceptés ou refusés par le patient dans une situation déterminée. Il peut être plus ou moins détaillé et constitue, avec les «directives » anticipées, la modalité d'extériorisation de la volonté du patient sur les soins projetés.

Quant à sa forme, le projet prévoit des règles de preuve visant à prouver l'authenticité du consentement du patient tout en envisageant d'autres manifestations de volonté. L'écrit est privilégié puisqu'il garantit la provenance de l'acte - et à ce titre est inscrit dans le dossier médical - mais ne remplace pas le processus réel de formation du consentement ${ }^{45}$.

\section{Les responsabilités posées : la prévision du déséquilibre}

Celui-ci nait d'un changement de circonstances, source d'imprévision. L'urgence médicale est visée mais aussi le désaccord et l'interruption d'un traitement médical en cours. Le projet de loi prend position en posant les responsabilités des parties afin de pallier l'incertitude que la disproportion fait courir.

S'agissant de l'urgence et du refus de soins non prévus ${ }^{46}$, les règles en matière d'intervention, d'abstention et d'interruption des traitements sont formulées au prix d'une adaptation du bénéfice escompté sur la santé du patient. Alors que cette évaluation se fait de façon strictement objective dans le premier cas, la qualité de vie et le bien-être psychologique du patient sont pris en compte pour discipliner le refus de soins qui met en œuvre «la conscience de sa propre mesure $»^{47}$.

Ainsi en cas d'urgence médicale, la décision incombe au médecin qui doit prêter assistance à la personne en danger. Ce devoir légal d'intervention est toutefois strictement encadré ${ }^{48}$ car il met directement en échec le principe de consensualita ${ }^{49}$.

Le praticien ne peut pas agir dans deux situations déterminées: en présence d'une volonté contraire du patient - exprimée au moment de l'intervention (hypothèse d'une personne consciente) ou précédemment, dans le cadre de «directives» anticipées ou d'une programmation des soins ${ }^{50}$; et lorsque son intervention serait contraire au respect dû à l'identité

\footnotetext{
${ }^{45}$ Projet, art. 5, al. 2.

${ }^{46}$ Projet, art. 14, al. 1 relatif à l'urgence médicale et art. 11 à 13 sur le refus de soins.

${ }^{47}$ P. ZATTI, Diritto e medicina, cit.

${ }^{48}$ Le domaine du professionnel de santé (en fonction de ses compétences, et seulement pour les soins nécessaires), la durée de l'intervention (le temps de l'urgence), son objectif (un traitement bénéfique),

${ }_{49}$ «Dès que la situation d'urgence prend fin, le principe de consensualità des soins s'applique à nouveau de plein droit »: Projet, art. 14, al. 4.

${ }^{50}$ Projet, art. 15.
} 
de la personne ${ }^{51}$. Ces limitations rejoignent les hypothèses du refus de soins, objet du chapitre IV, où l'appréciation du caractère approprié du traitement médical incombe au patient qui dispose d'un droit fondamental, en miroir du principe de l'habeas corpus.

En effet, face à un refus - seconde hypothèse - le médecin doit s'abstenir de toute intervention, fût-elle bénéfique. Du point de vue du patient, ce refus peut consister à interrompre des soins auparavant consentis, ou bien à ne pas donner son accord à un nouveau traitement, même vital ${ }^{52}$. Quant au devoir d'abstention du médecin, il peut s'agir soit d'une omission (arrêt du traitement) soit d'une action (débrancher un appareil) : dans les deux cas, l'abstention consacre la réalisation du droit fondamental au refus de soins (art. 32, al. 2 de la Constitution) ${ }^{53}$ où la responsabilisation du patient s'entend du reflet objectif de son auto-détermination.

En raison de la gravité des conséquences liées au refus, le projet de loi prévoit en faveur du malade des mesures de protection: l'incitation à la réflexion et le soutien psychologique. Enfin une obligation renforcée d'information est mise à la charge du médecin ${ }^{54}$ et la collaboration de toute l'équipe de soins est requise ${ }^{55}$.

Outre les conditions d'exercice du droit au refus, le texte s'attache à en préciser les effets. S'agissant du médecin, un principe d'irresponsabilité $e^{56}$ et d'immunité de toute sanction est posé - tant dans l'hypothèse de reprise que celle d'accélération du processus naturel de mort - précédé de l'obligation

\footnotetext{
${ }^{51}$ Projet, art. 14, al. 3 dans l'hypothèse où il n'est pas possible de reconstruire la volonté du malade.

${ }^{52}$ Parmi ceux-ci sont, en particulier, visées la fonction cardiovasculaire et respiratoire, ainsi que l'alimentation et l'hydratation artificielle. À noter que, dans sa version définitive l'art. 2 de la proposition française considère, de même, que « La nutrition et l'hydratation artificielles constituent des traitements qui peuvent être arrêtés conformément au premier alinéa du présent article » (obstination déraisonnable, inutilité). L'hydratation artificielle n'est donc pas un « soin qui peut être maintenu jusqu'au décès ». Le renoncement à un support vital n'est pas un acte d'euthanasie passive car il n'implique pas l'intention de donner la mort mais de ne plus s'opposer à l'évolution naturelle de la maladie, v. adps p. 17. Enfin 1' «assistance médicalisée » qui s'apparente au suicide assisté n'est a fortiori pas envisagée dans le projet italien.

${ }_{53}$ «Nul ne peut être contraint à un traitement sanitaire déterminé, si ce n'est par une disposition de la loi. La loi ne peut, en aucun cas, violer les limites imposées par le respect de la personne humaine ». V. P. ZATTI, Premesse e criteri per un diritto, cit. A fortiori il ne s'agit donc pas d'euthanasie passive puisque le projet italien ne fait aucune référence au caractère désespéré de la situation - qui implique un jugement médical - et envisage l'arrêt exclusivement par rapport au principe de dignité et au droit à l'autodétermination du malade.

${ }^{54}$ En vertu de 1'art. 11, al. 2 du projet, le médecin est tenu de rendre « conscient » le malade des conséquences de sa décision.

${ }^{55}$ L'art. 13 du projet précise que les dispositions en matière de refus de soins (chap. IV) s'appliquent également aux professionnels de santé qui collaborent avec le médecin.

${ }^{56} \mathrm{~L}$ 'art. 11 , al. 4 , du projet se réfère à l'art. 51 du Code pénal selon lequel l'exercice d'un droit ou l'exécution d'un devoir imposé par la loi ou tout ordre émanant de l'autorité publique n'est pas pénalement répréhensible.
} 
de «rechercher ${ }^{57}$ le consentement du patient qu'il ait pour objet le commencement ou la poursuite des soins.

La prévision d'une clause de conscience ${ }^{58}$ autorise le praticien à ne pas exécuter la demande d'interruption des soins, si celle-ci s'avère contraire à ses propres conceptions éthiques. Dans ce cas, et parce qu'il fait valoir son autonomie professionnelle et morale, il doit avertir l'établissement et prêter assistance au patient le temps de sa prise en charge par un autre médecin ${ }^{59}$.

Quant au patient, la sauvegarde de sa dignité ${ }^{60}$ est envisagée sous deux profils, spéculaires des obligations du professionnel de santé. Le premier concerne le droit à des soins " autres que curatifs », c'est-à-dire à des actes élémentaires d'attention qu'exige le respect dû à sa personne (toilette, offre d'aliment et d'eau par voie buccale) et que corrobore le principe d'interdiction de l'abandon thérapeutique. Ne sont pas visés les soins palliatifs dont l'accès est garanti par la loi 38/2010 .

Le deuxième droit est relatif à la mise en œuvre du refus : le projet de loi pose l'obligation, à charge des établissements de prédisposer un protocole de soins appropriés. Ce devoir entend faire échec à certaines pratiques tendancieuses que le Conseil d'État italien a fermement condamnées : les structures sanitaires « ne peuvent opposer à l'exercice d'un droit fondamental, une conception qui leur serait propre de la prestation de soins $»^{62}$.

\section{LES DISPOSITIONS ANTICIPÉES DE TRAITEMENT ${ }^{63}$ : ENTRE DROIT ET RÉALITÉ}

Le projet italien utilise le mot disposizioni (et non directive) par renvoi au droit subjectif du patient de choisir « le sort de son corps », d'en disposer. À nouveau et a fortiori, la liberté personnelle est valorisée excluant tout recours à l'idée d'imposition propre à la norme juridique : la sanction est absente, la force du texte résidant en sa capacité à se saisir d'une situation

\footnotetext{
${ }^{57}$ L'emploi du verbe «ricercare » met l'accent sur le caractère approfondi, malaisé du processus qui porte à la manifestation de la volonté du patient.

${ }^{58}$ Cette clause est envisagée dans le texte français à propos de la sédation terminale.

${ }^{59}$ Projet, art. 12, al. 1.

${ }^{60}$ Projet, art. 12, al. 2 ; de même l'al. 3 se réfère, par renvoi à l'art. 2, aux choix identitaires du patient comme déterminants de la relation de soins.

${ }^{61}$ Supra note (8)

${ }^{62}$ Consiglio di stato, sez. III, 02.9.2014, n. 4460 et la note de P. BENCIOLINI, « Obiezione di coscienza? ", Nuova Giurisprudenza Civile Commentata, 2015, II, p. 3. Cette obligation met un terme à une pratique condamnable dont a été le siège l'affaire Englaro, v. R. FERRARA, « Il caso Englaro innanzi al Consiglio di stato », ibid., p. 9. De même en France le cas Lambert illustre les difficultés de mise en œuvre du droit à laisser mourir.

${ }^{63}$ Elles sont l'objet des sections II, III et IV, Chapitre IV du projet.
} 
individuelle qui, par hypothèse, s'inscrit dans l'instant et non dans la duré $^{64}$. La règle doit «apprendre et se mettre à l'écoute» d'une seule personne ${ }^{65}$ pour encadrer le temps présent, les «moments de retour sur soi $»^{66}$, c'est ce que recouvre l'agilité du droit. Des critères de validité et d'efficacité des dispositions anticipées doivent alors être posés.

\section{A. - Validité : les critères de fond et de forme}

\section{Le fond: le respect de la volonté et de l'identité de la personne}

Les dispositions anticipées sont «les déclarations qu'une personne, en prévision de son incapacité à se déterminer ou à manifester sa volonté, exprime son consentement ou son refus sur des choix thérapeutiques, y compris la nutrition et l'hydratation artificielles ${ }^{67}$, et dicte ses orientations ou manifeste ses préférences sur les soins et l'attention (accudimento) qu'elle veut recevoir ou qu'elle entend exclure $\rangle^{68}$.

S'agissant de décisions de fin de vie, le projet prévoit un système renforcé de garanties pour protéger non seulement la volonté du patient mais aussi son identité, en tant que personne ${ }^{69}$, l'idée étant que le droit à la vie est indissociable de la dignité du vécu ${ }^{70}$. Par conséquent, une société « humaine » ne peut refuser de dispenser les mesures indispensables à la sauvegarde de la dignité, au risque de désavouer la volonté du disposant ${ }^{71}$ (la nutrition et l'hydratation artificielles sont considérées comme des traitements médicaux et ne sont donc pas visées). Logiquement la grande flexibilité des critères adoptée à propos du consentement aux soins du patient se retrouve a fortiori dans ce domaine.

Les dispositions de volonté du patient peuvent ainsi avoir un contenu très varié : le choix d'une thérapie particulière pour une situation déterminée ou bien, au contraire, la prévision de critères très généraux relatifs à des modalités d'intervention risqués ou impliquant des conditions de vie inacceptables.

${ }^{64}$ Sur la discordance des temps (chronos et kairos), v. C. CHAINAIS, cit.

${ }^{65} \mathrm{~V}$. la relation introductive au projet cit. supra note (1).

${ }^{66}$ V. C. CHAINAIS, cit.

${ }^{67} \mathrm{~V}$. supra note $(50)$

${ }^{68}$ Art. 18.

${ }^{69} \mathrm{Il} \mathrm{s}$ 'agit de la principale différence avec la programmation des soins.

${ }^{70}$ Premesse e criteri, cit.

${ }^{71}$ Art. 19, al. 2 ; le projet applique les principes de dignité énoncés aux articles 1,2 et 3 al. 2 et ne porte pas préjudice au droit au refus de soins et de traitements garanti par les articles 11, 18 et 19. 
Le recours à une «déclaration d'identité ${ }^{72}$ est également envisagé pour intégrer ou même suppléer la manifestation de volonté du malade, envisagé comme membre de la société humaine. Il s'agit d'une notion très large fondée sur l'autodétermination ${ }^{73}$ qui renvoie à l'idée d'ipséité, l'identité narrative qui $《$ s'inscrit dans le temps $»^{74}$. Sont ainsi visés les « convictions, préférences, croyances, penchants » sur la maladie et la fin de vie en mesure de refléter, de façon certaine, la « conception que la personne se fait d'elle-même et de l'existence » et qui trouveraient support dans des déclarations publiques, lettres, signes religieux, ... toute indication susceptible de valoriser l'individualité, en tant que telle, et que l'acte juridique - les dispositions de volonté - ne peut exprimer.

Dans le même ordre d'idées s'inscrit le recours à un intermédiaire personne de confiance ou curateur - pour reconstituer l'histoire de la personne. À cette fin, le projet favorise, en relation avec les circonstances, le respect tant des dispositions de volonté que des signes identitaires de la personne $\mathrm{e}^{75}$. Leur praticabilité au regard du traitement et de l'assistance médicale ${ }^{76}$, est la seule condition posée à leur mise en œuvre.

En général, les moyens utilisés au service de la recherche identitaire visent à imprimer, dans le temps, la marque d'une personne. La démarche n'est pas aisée, témoignant de la difficulté, en raison des "croisements » avec la psychiatrie et la philosophie ${ }^{77}$, à donner un contenu juridique à l'autonomie.

\section{La forme: les exigences minimum}

La liberté de la forme est consacrée : la volonté du patient peut se manifester par écrit, oralement ou par toute " procédure adaptée aux moyens d'expression du disposant » à condition qu'elle soit recueillie par le médecin responsable des soins (témoignage retranscrit dans le dossier médical, enregistrement audio sous pli daté et signé, ...) ou documentée dans un enregistrement vidéo ayant date certaine ${ }^{78}$. Toutes les expressions sont visées puisqu'il s'agit de faciliter la diversité d'écoute individuelle. Des exigences minimum de sécurité juridique concernant la provenance, le contenu et la date des dispositions anticipées sont posées ${ }^{79}$.

${ }^{72}$ V. Rapport introductif au projet de loi, cit., p. 4.

${ }^{3}$ P. ZATTI, Premesse e criteri, cit.

${ }^{74} \mathrm{C}$. CHAINAIS, cit. par référence à P. RICEUR.

${ }^{75}$ Projet, art. 20.

${ }^{76}$ Projet, art. 19, al. 3.

${ }^{77}$ B. MORIZOT, cit., p. 21.

${ }^{78}$ Outre cette dernière modalité, l'art. 23 du projet vise l'acte publique, l'acte sous seing privé remis au médecin, l'écrit signé et daté et les déclarations orales.

${ }^{79}$ Les établissements de soins/médecins généralistes sont tenus d'informer les patients sur leur droit à disposer de façon anticipée ; cette obligation s'étend aux mairies qui, à l'occasion d'un 
L'intervention d'un tiers, le notaire ou toute autre personne habilitée à recevoir et conserver les dispositions, n'est prévue que si l'intéressé luimême en fait la demande. En cas d'incapacité, les éventuelles dispositions contenues dans l'acte de désignation du curateur valent comme dispositions anticipées de traitement ${ }^{80}$.

La révocation des dispositions est entièrement libre et peut avoir lieu à tout moment, à charge pour celui qui la recueille - hypothèse d'un intermédiaire - d'en informer de façon urgente le médecin responsable. Aucune durée de validité n'est prévue.

\section{B. - L'efficacité : la problématique de l'opposabilité}

Le but consiste bien évidemment à exécuter les dispositions anticipées et le projet, attentif à nouveau, à la question de la temporalité élabore des pistes pour résoudre le décalage - éventuel - entre la prévision et la mise en œuvre. Afin de refléter au mieux la réalité du vécu, deux parcours sont envisageables.

À l'égard d'une situation en cours, le professionnel de santé (ou quiconque assiste le malade) est tenu au « respect total des dispositions anticipées » : le projet n'a pas recours à l'instrument de l'opponibilità dont la fonction est de déterminer l'efficacité d'un acte dont la connaissance est malaisée ${ }^{81}$; en la circonstance, il ne s'agit pas de résoudre un doute que le temps aurait créé. Le caractère actuel de la manifestation de volonté du malade $^{82}$ et le devoir pour le médecin de la respecter, sont suffisants à satisfaire une application personnalisée et partant le principe de sécurité juridique.

À l'égard d'une application distante dans le temps, le risque d'une décision disproportionnée ${ }^{83}$ est appréhendé à travers deux moyens: la recherche de la signification réelle des dispositions et la mise en œuvre «renforcée » du principe de collégialité même si le médecin reste seul responsable de la décision. Ce dernier est tenu, en accord avec la personne de confiance, d'interpréter ${ }^{84}$ et de mettre en pratique les dispositions anticipées. En cas de désaccord persistant, la saisine du juge des tutelles est

renouvellement ou d'une demande de documents d'identité, sont tenues d'informer leur résidents, v. art. 23, al. 3, a) du projet.

${ }^{80}$ Projet, art. 24.

${ }^{81}$ P. ZATTI, V. COLUSSI, Lineamenti di diritto privato, Cedam, 2013 p. 1013.

${ }^{82}$ Ainsi que des désirs, convictions et penchants visés à l'art. 21, al. 1 du projet.

${ }^{83} \mathrm{Ce}$ point rejoint le débat français sur le caractère " manifestement inapproprié (des directives) ou non conformes à la situation médicale du patient ", v. art. 8, al. 4, proposition définitivement adoptée.

${ }^{84}$ D'où la recherche de leur signification réelle : P. ZATTI, Premesse e criteri, cit. 
prévue. Le principe de sécurité juridique joue au profit du médecin « respectueux » des dispositions dans la mesure où sa responsabilité ne peut être engagée ${ }^{85}$.

Le projet instaure un espace décisionnel pour vérifier l'efficacité des dispositions eu égard aux circonstances de fin de vie et réintégrer, le cas échéant, des expériences essentielles à la réalisation de $\mathrm{soi}^{86}$; ce contrôle de concordance est perçu comme le moyen le plus efficace pour sauvegarder la dignité de la personne ${ }^{87}$ et rappelle que le «caractère manifestement inapproprié » des directives, tel que visé dans la proposition française, n'est pas aisé à déterminer ${ }^{88}$.

L'emploi de concepts juridiques se fait parcimonieux à mesure que la décision médicale met en œuvre le seul principe de dignité. Ainsi - et exception faite d'un refus explicite sur un traitement qui s'impose au médecin - c'est un devoir d'adapter la gestion médicale aux «lignes directrices » des dispositions qui est institué. Il ne s'agit pas pour autant de « tenir compte» des «directives» (art. 9 Convention de Oviedo et art. 8 proposition française ${ }^{89}$ ) mais de les rendre praticables - seule limite à la pleine efficacité des dispositions - en harmonie avec le droit à l'autodétermination et le respect de l'identité de la personne ${ }^{90}$, vrais supports temporels de la fin de vie.

Le projet italien, s'il est conforme aux sources européennes et rejoint sur l'essentiel la proposition française ${ }^{91}$, les dépasse pourtant par la subjectivité qui guide tout le processus décisionnel de la fin de vie. Il peut ainsi paraitre aux confins du droit tant la temporalité de la situation est difficilement saisissable par le législateur ${ }^{92}$. Pour pallier cet inconvénient, le projet prend appui sur la relation de soins à laquelle il imprime une certaine

${ }^{85} \mathrm{Il}$ est de même exonéré de toute sanction : projet, art. 22.

${ }^{86}$ Le respect est d'autant plus pressant qu'aucune manifestation de volonté n'est intervenue (incapacité, inconscience, absence de dispositions anticipées) et pour cette raison, l'euthanasie pourrait s'avérer réductrice: A. FORNES, P. BONHOMME, cit., p. 20) d'autant plus que les demandes d'euthanasie émanent le plus souvent de l'entourage que du patient lui-même, v. G. DARCOURT, cit.

${ }^{87}$ Projet, art. 21, al. 3.

${ }^{88}$ V. l'art. 8 de la proposition française (directives anticipées).

${ }^{89}$ Amendement 67 rect. bis relatif à l'art. 8 de la proposition de loi.

${ }^{90}$ P. ZATTI, Premesse e criteri cit.

${ }^{91}$ Bien que le projet français se focalise sur la sédation en phase terminale, l'opposabilité des directives anticipées et le développement des soins palliatifs, il n'est pas douteux qu'il a pour objectif d'accorder davantage de droits au patient en se fondant sur leur volonté et qu'il s'agit d'un processus vers lequel tendent tous les législateurs nationaux.

${ }^{92}$ C. CHAINAIS, cit. 
normativité ${ }^{33}$, dès lors que celle-ci s'inscrit dans la durée, tirée des droits fondamentaux. À l'égard de situations non prévisibles ou vis-à-vis desquelles est prôné un retour à l'ordre naturel, la mise en œuvre du droit au refus de soins et de son corolaire - l'irresponsabilité du médecin - est appréhendée principalement, hors du schéma juridique, sur la base de critères tenant à l'harmonie du vécu. Là réside sans doute la valeur du pari transalpin qui mise sur la capacité d'écoute individuelle de la règle pour accompagner la personne, lui restituer « son » temps.

${ }^{93}$ Par exemple le recours à l'acte juridique dans la programmation des soins, aux règles de forme de ces mêmes actes, à la non imputabilité du médecin qui met en œuvre les dispositions anticipées du patient. 